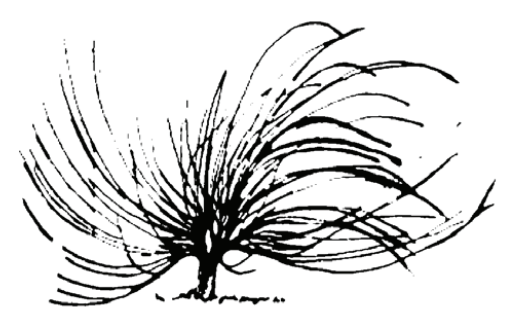

\title{
Comunidades Virtuales de Aprendizaje: Una Herramienta para Fomentar la Inclusión en la Enseñanza del Inglés desde la Contextualización Curricular
}

\author{
Karla Jiménez Porras ${ }^{1}$ \\ Centro Educativo Niño Jesús de Praga \\ Alajuela, Costa Rica \\ karla.jimenezp@gmail.com \\ Vanessa Jiménez Porras ${ }^{2}$ \\ Universidad Técnica Nacional \\ Alajuela, Costa Rica \\ vane.jp8@gmail.com
}

\section{Resumen}

El aprendizaje y la enseñanza del inglés han sido considerados una prioridad en el currículo nacional por décadas, a partir de los cambios económicos, políticos y culturales de la coyuntura global actual. No obstante, los métodos pedagógicos tradicionales para la enseñanza de lenguas extranjeras se imponen en las aulas y limitan el desarrollo de competencias comunicativas en los estudiantes, lo cual se intensifica cuando los estudiantes con algún tipo de necesidad educativa especial ingresan al sistema

\section{(c) () $\Theta$}

Recibido: 9 de mayo de 2015-Aprobado: 26 de noviembre de 2015

1 Coordinadora del Departamento de Inglés y docente, Centro Educativo Niño Jesús de Praga. Alajuela, Costa Rica. Licenciada en Administración Educativa, Universidad Estatal a Distancia. Cursando la Maestría en Educación con énfasis en el aprendizaje del inglés, Universidad Nacional. Heredia, Costa Rica.

2 Profesora de cursos libres de inglés. Universidad Técnica Nacional. Alajuela, Costa Rica. Licenciada en Docencia, Universidad de San José. Cursando la Maestría en Educación con énfasis en el aprendizaje del inglés. Universidad Nacional, Heredia. 
educativo. Esto hace necesario la implementación de una educación inclusiva para alcanzar el éxito de todos. Una comunidad virtual de aprendizaje profesional para docentes en lengua extranjera representa una alternativa viable para implementar prácticas inclusivas en el aula.

Palabras clave: enseñanza, formación profesional, comunidades de aprendizaje, lengua extranjera, inclusión.

\begin{abstract}
In Costa Rica, English teaching and learning have been considered a priority for decades, in order to help students face life and work situations which require an average command of English, with the desire that this preparation will allow them to participate actively into the challenges of the global economy. However, traditional teaching methods for language learning are implemented in the classrooms, limiting the acquisition of communicative skills in the students, which is more noticeable when those with Special Learning Needs walk into the regular classrooms. Inclusive practices become a must to promote every student's success. A virtual learning community for teachers becomes a feasible tool to offer teachers with training to cater for the needs of those students.
\end{abstract}

Keywords: teaching and learning, professional development, virtual learning communities, English as a Foreign Language, inclusion

\title{
Introducción
}

El sistema educativo costarricense se ha comprometido durante años a la universalización de la educación. Los gobiernos y las autoridades educativas deben asegurarse de que cada ciudadano reciba igualdad de oportunidades para participar efectivamente en los procesos educativos. Para atender las demandas de la educación inclusiva, existe una aceptación general acerca de que los docentes deben ser entrenados para enseñar efectivamente en instituciones donde existen estudiantes con cierta variedad de necesidades educativas especiales (NEE). 
Por otra parte, el aprendizaje y la enseñanza del inglés han sido considerados una prioridad nacional por décadas. La lengua inglesa se ha convertido en predominante a partir de los cambios económicos, políticos y culturales. "Se trata de la herramienta lingüística y comunicativa utilizada en los procesos actuales de expansión económica, tecnológica, científica y cultural" (Paz y Suárez, 2010, p. 9). Los estudiantes están expuestos al inglés en diferentes actividades de su vida diaria, por lo que el aprendizaje de este idioma, adquiere gran relevancia como herramienta de comunicación.

En muchos países, incluido Costa Rica, el enfoque comunicativo ha formado parte de las bases teóricas que sustentan los programas de la enseñanza de inglés como lengua extranjera desde la década de 1970. En 2005, bajo el lema "Relanzamiento de la educación costarricense", el Ministerio de Educación Pública (MEP) rediseñó los programas de estudio de la educación secundaria, para el ciclo diversificado. Según Domian, Fonseca, Lara y Rodríguez (2011), el Programa de Estudio 2005 Inglés Educación Diversificada se elaboró con el objetivo de desarrollar la competencia comunicativa de los estudiantes y procura "el fomento del aprendizaje de una lengua extranjera que permita aumentar el intercambio con otras culturas y, a partir de allí, entender mejor la propia" (p. 13). Además, considera las necesidades y los estilos de aprendizaje para ofrecer a los estudiantes diversas oportunidades que les permitan un uso significativo de la lengua.

Aunque gran parte de la población costarricense admite la necesidad de aprender inglés como medio para comunicarse en el mundo globalizado, los métodos pedagógicos tradicionales para la enseñanza de lenguas extranjeras se imponen en las aulas y limitan el desarrollo de competencias comunicativas. Aún más, cuando estudiantes con algún tipo de NEE ingresan al aula regular de inglés, los docentes enfrentan una situación para la cual no han sido entrenados, ni en la universidad ni en la institución para la cual laboran.

Muy frecuentemente, se espera que los docentes implementen una gran gama de prácticas educativas para alcanzar estándares comunicativos en la adquisición de una lengua extranjera. Sin embargo, la mayoría de las veces los docentes de inglés no se sienten preparados para enfrentar la amplia diversidad de NEE que encuentran en sus aulas. También es cierto que, durante sus años de formación universitaria, no existe una verdadera práctica acerca de cómo las metodologías que se espera que implementen en sus aulas apoyen el aprendizaje 
comunicativo de estudiantes de todas las habilidades. La mayoría de docentes de inglés desconocen estrategias efectivas o técnicas empleadas para enseñar inglés en forma significativa a estudiantes con NEE.

\section{Justificación}

Es importante concebir la práctica profesional docente como práctica social, esto es, en su vinculación con los procesos sociales más significativos. Para el docente reflexivo y analítico, las NEE de los estudiantes son aspectos significativos a considerar al planear estrategias de aprendizaje en el aula, considerando las individualidades de sus estudiantes, las demandas del currículo, y las de su entorno económico y social.

El currículo se concibe como un proceso, más que un orden de acciones previamente establecidas. Debe ser el docente, en un análisis crítico de la realidad, quien permee dicho currículo de humanismo y calidad por medio de un empoderamiento real y una práctica pedagógica participativa e integrada con las demandas de los estudiantes.

Un diseño curricular de lenguas extranjeras válido, útil y eficaz es un instrumento indefinidamente perfectible y en constante cambio, cuyo uso por los docentes no se limita (o no debería limitarse) a una aplicación automática de acciones prescritas por otros. Un buen diseño curricular no ofrece soluciones acabadas o definitivas para cada una de las situaciones, sino que proporciona elementos útiles a los docentes para que se puedan elaborar, en cada caso específico, las soluciones más adecuadas en función de las circunstancias concretas en las que tiene lugar la práctica educativa. En inglés, estos elementos son flexibles, incorporan elementos culturales y curriculares, tomando en cuenta las NEE de los estudiantes en un contexto particular.

No basta contar con diseños curriculares cuidadosamente elaborados, científicamente fundamentados y profesionalmente contrastados. Es necesario impulsar su desarrollo y convertirlos en instrumentos de trabajo, indagación y perfeccionamiento por parte de la comunidad docente, la cual debe asumir un papel activo en la innovación pedagógica. Así pues, la finalidad que debe perseguir todo diseño curricular es estimular la contextualización, la creatividad y adecuarse a las particularidades de las aulas.

Una comunidad virtual de aprendizaje profesional, diseñada y construida para el desarrollo profesional del docente de inglés, surge 
como una alternativa viable para incorporar, mejorar e innovar diversos elementos del currículo. Por ejemplo, que los sujetos sean activos en el proceso, que se tome en cuenta tanto las necesidades económicas del entorno particular, como los requerimientos particulares para acceder a dichos aprendizajes e incorporarse al entorno laboral; así como la flexibilidad en las experiencias de aprendizaje y evaluación con el fin de que los aprendizajes esperados sean significativos, permanentes y contextualizados.

Una de las tareas de los docentes de cualquier nivel educativo es la de planificar el currículo en el ámbito de la instrucción. Esta es una actividad compleja, que pasa por el análisis de una serie de variables interrelacionadas (objetivos, contenidos, actividades de aprendizaje, recursos y estrategias de evaluación) que buscan alcanzar un fin específico. Elaborar un diseño curricular de lenguas extranjeras supone un proceso de toma de decisiones que el profesor debe asumir como parte fundamental de su labor, para adecuar su intervención docente a las necesidades reales de aplicación en el aula.

Si bien es cierto, se cuenta con un programa nacional de inglés que debe ser aplicado, se tienen unos objetivos que se requiere que sean alcanzados y el medio socioeconómico y cultural demanda de los estudiantes y docentes ciertas habilidades en el proceso, las cuales no podrán ser alcanzadas si los docentes no cuentan con criticidad y desenvolvimiento en el momento de poner el currículo en práctica. Las NEE son una realidad en nuestras aulas regulares de educación, pero ellas no deben ser una limitante para que todos los estudiantes logren alcanzar el éxito en el aprendizaje de una lengua extranjera. Este es un compromiso que debe ser asumido por los docentes, los administradores educativos $y$ las instituciones de enseñanza en forma articulada.

Frente a la existencia de un currículo nacional uniforme y prescriptivo, frecuentemente, ajeno a la participación activa de los profesores y en el que se muestra una descontextualización en relación con las necesidades de los entornos particulares, un segundo modelo, como el que se promueve la creación de comunidades virtuales, postularía un tipo de currículo diferente. Sería uno abierto y flexible, en el que los docentes se convierten en auténticos protagonistas de su quehacer educativo, asumiendo el reto de que supone la adecuación de las actividades de aprendizaje y estrategias de evaluación a las NEE particulares del estudiantado y a la realidad contextual en que se desarrolla su tarea. 
Muchos de los profesores de inglés en servicio en una institución educativa en Alajuela han procurado apoyar el aprendizaje de sus estudiantes con NEE de distintas maneras, aunque carezcan de entrenamiento o capacitación para hacerlo. Prueba de ello, son algunas de estas estrategias que han sido exitosas, y los estudiantes con NEE han logrado avanzar en el aprendizaje de la lengua extranjera, aunque no en el ritmo esperado por los docentes o por los mismos estudiantes.

Los profesores de inglés como segunda lengua de dicha institución han expresado la necesidad de contar con una herramienta a la cual puedan recurrir, que sea accesible y útil para la demandante vida laboral y profesional que enfrentan. Deberá además brindarles la oportunidad de crecer profesionalmente, escoger entre varias estrategias de enseñanza, las cuales se puedan adaptar para crear su propio marco de referencia, de forma tal, que ellos puedan decidir cuáles son las técnicas idóneas para apoyar a los estudiantes según sus características individuales, y así mejorar la inclusión y las habilidades comunicativas del alumnado.

El diseño y la construcción de una comunidad virtual de aprendizaje (CVA) se presenta como una herramienta para el crecimiento profesional de los docentes de inglés, que laboran en la institución, por diferentes razones. La literatura educativa estipula que una CVA puede servir como medio para mejorar el rendimiento académico en el sistema escolar, que contribuye a desarrollar y crear valores y visión compartida; así como la responsabilidad colectiva del profesorado por el aprendizaje de los estudiantes. Además, estimula la colaboración; el aprendizaje profesional, individual y colectivo; la investigación profesional reflexiva; el sentimiento de pertenencia a una comunidad; genera confianza, respeto y apoyo mutuos, entre otros (Bolívar Ruano, s. f.; Lewin, 1999; Lloyd y Duncan, 2010; Saborío, s. f.).

Los factores tiempo y distancia podrían convertirse en limitaciones para asistir a un taller de capacitación. Por esta razón, una CVA se convierte en un espacio muy útil para que los profesores compartan y aprendan de sus propias experiencias.

El crear una CVA es un proyecto complejo, ya que al estar basado en la red, no puede estar limitado a cierto espacio geográfico o población. Sin embargo, el sitio web está dirigido a esta población docente en particular, sus necesidades de formación en esta área, su contexto, y las NEE más frecuentes diagnosticadas en su población estudiantil, las cuales han sido reportadas por el Comité de Apoyo Educativo. 
El sitio web actualmente se encuentra en etapa de construcción y aplicación, con el objetivo de que se convierta en una verdadera CVA para mejorar el desarrollo profesional de los docentes de inglés en NEE y prácticas inclusivas. Al finalizar el curso lectivo 2015, se espera evaluar, en conjunto con el profesorado meta, la efectividad de la participación y la información ofrecida, así como su impacto en las prácticas de aula. La página puede ser accesada en la dirección electrónica www. teachersforinclusion.com

\section{Diagnóstico}

Los problemas económicos de Costa Rica en las últimas décadas han demorado los esfuerzos por proteger los derechos humanos de las personas con NEE. De acuerdo con el sitio red del Instituto Middlebury, la población con discapacidad se encuentra entre los sectores más excluidos de la sociedad costarricense. A pesar de que existen leyes para proteger a las personas con capacidades diferentes, "el gobierno no ha logrado crear las condiciones necesarias para la mayoría de las personas con discapacidad en Costa Rica que les posibiliten ejercer completamente sus derechos como ciudadanos" (IDRM, 2004).

Un reporte elaborado por el Departamento de Educación Especial del Ministerio de Educación Pública (s.f.), ofreció una visión general de la educación para personas con discapacidad. Los estudiantes con NEE abarcan alrededor del $10.13 \%$ del número global de la población estudiantil del país. Existen aproximadamente 95000 estudiantes con NEE. La mayoría acude a aulas convencionales, según este documento.

Los datos anteriores sugieren que la cantidad de población con NEE es importante dentro de nuestras aulas, e ignorar estas condiciones no solucionará los inconvenientes que se están presentando con estos estudiantes, en términos de las limitaciones que presentan en la adquisición de habilidades comunicativas. Ciertas acciones se pueden llevar a cabo por los docentes, tales como: reflexionar sobre estudios de caso, analizar su propia práctica y compartir conocimientos. Estas se consideran las más adecuadas para preparar a los docentes para la educación inclusiva (Wade, 2000), lo cual les permite conocer las necesidades y preocupaciones de sus estudiantes, así como desarrollar una adecuada comprensión, actitud y experiencia al resolver problemas similares en escenarios inclusivos. Wade (2000) afirma: 
Enseñar Estudiantes Diversos en Aulas Inclusivas es un curso desarrollado en la Universidad de Utah para abordar temas de diversidad y educación inclusiva. Incluye no solamente discusiones relacionadas con la educación inclusiva, sino también posiciones, planes de estudio, enfoques educativos y prácticas de evaluación que promueven el aprendizaje para todos los estudiantes, que de otra forma podrían fracasar ... Además, este curso investiga convenios colaborativos que puedan proveer el conocimiento y los recursos necesarios para el éxito de los esfuerzos inclusivos (p. 25).

Las universidades o instituciones educativas costarricenses pueden estudiar experiencias como la mencionada anteriormente, junto con sus propios métodos reflexivos y prácticas de desarrollo docente, para atender las necesidades de su contexto y población diversa. El curso de capacitación mencionado por Wade (2000) se enfoca en diferentes aspectos:

- Profesionalismo y participación.

- Escritura de un portafolio de estudio de casos.

- Desarrollo una comunidad de aprendizaje profesional.

Los debates, la discusión de videos y la construcción de conocimiento colectivo son aspectos importantes para tomar en consideración en esta propuesta. Aunque no se han localizado iniciativas similares para docentes de inglés en Costa Rica, se espera que la reflexión entre profesionales de la educación pueda llevar a propuestas semejantes, contextualizadas y adaptadas a las necesidades de los docentes costarricenses.

\section{Problema}

Las experiencias previas de los docentes permean fuertemente sus creencias y concepciones sobre el proceso de enseñanza. Por lo tanto, la capacitación dirigida a educadores practicantes (también conocida como desarrollo profesional) debe ser tomada en consideración cuando la formación universitaria no ha provisto a los estudiantes de educación con el conocimiento necesario para implementar prácticas inclusivas en las aulas de idiomas. 
Los docentes de inglés como lengua extranjera que laboran para instituciones del Ministerio de Educación Pública (MEP) dependen de las recomendaciones del Comité de Apoyo Educativo para atender a los estudiantes con NEE. Estas se limitan a sugerencias en términos de adecuaciones curriculares para el trabajo de aula y la evaluación, las cuales no resuelven realmente las necesidades de los estudiantes de aprender a comunicarse en forma efectiva en la lengua extranjera ni aseguran un ambiente inclusivo en las aulas de inglés. Esta situación se convierte en un vacío entre lo que los docentes conocen y lo que se requiere que implementen en las aulas, mientras que estos estudiantes enfrentan mayores dificultades en la adquisición de la lengua extranjera que los demás aprendientes de la clase

De acuerdo con la administradora del centro en el que se trabaja esta propuesta, la gestión educativa ha detectado una necesidad de capacitación entre los docentes de inglés para que sean capaces de identificar, atender y evaluar en forma inclusiva a estudiantes con NEE. Esto de acuerdo con los datos aportados por el Comité de Apoyo Educativo, en relación con el rendimiento académico en el área de inglés de los estudiantes que presentan NEE durante los últimos tres años, el cual ha sido de moderadamente bueno a regular (E. Sánchez, comunicación personal, 15 de febrero de 2015).

De esta forma, el sitio web que se ha propuesto se ofrece como una oportunidad en forma de esfuerzo colaborativo, que busca la discusión y la evaluación formativa. Esta CVA será elaborada por y para docentes de inglés, con el fin de compartir conocimientos, experiencias previas y estrategias metodológicas con el fin de ajustar las prácticas docentes para atender mejor la diversidad. De esta forma, tomará en cuenta también el contexto y los problemas particulares que se presentan a los docentes que pertenecen a esta comunidad.

\section{Argumentación Teórica}

\section{Desarrollo profesional docente}

Muy frecuentemente, se espera que los docentes implementen una creciente gama de estrategias metodológicas para alcanzar los estándares comunicativos en la adquisición de una lengua extranjera. Sin embargo, durante su preparación universitaria, en Costa Rica, pareciera 
no existir una intención clara de demostrar cómo dichas prácticas y estrategias se relacionan o apoyan el aprendizaje significativo.

Richards y Farrell (2005) plantean que, más que ver el desarrollo de estrategias de enseñanza como el conocimiento de principios generales que han sido determinados por otros, la adquisición de pericia docente se percibe como un proceso que involucra al educador en la construcción activa de una teoría educativa personal, aplicable en su contexto. No hay capacitaciones o libros que "enseñen a enseñar"; sin embargo, la experiencia profesional y el consejo entre colegas pueden ser muy beneficiosos para compartir información, construir conocimiento colectivo y crear teoría pedagógica.

Por otra parte, lo que los docentes hacen está determinado por lo que piensan, y sus creencias actúan como un filtro a través del cual realizan sus decisiones instruccionales. La CVA busca estimular el aprendizaje entre sus participantes, no desde teorías predeterminadas sino desde los mismos participantes y sus propias prácticas educativas, teorías compartidas, contexto, participación de expertos y otras fuentes.

Una vez que los docentes son conscientes de la responsabilidad que tienen al pensar y teorizar su propia práctica, pueden enfocarse mejor en la acción e involucrarse en ajustar y evaluar su propio rendimiento. Existe también la necesidad de cerrar el vacío entre investigación, teoría y práctica. Esto podría lograrse al observar hasta qué grado los docentes consideran relevantes para su propia práctica educativa las estrategias sugeridas por sus colegas.

\section{Comunidades Virtuales de Aprendizaje (CVA)}

La literatura educativa ha ayudado a promover un nuevo conjunto de prácticas de desarrollo profesional, tales como la investigación docente, la investigación-acción participativa, la colaboración profesional y las comunidades virtuales de aprendizaje (CVA) para brindar a los educadores la oportunidad de alcanzar las exigentes expectativas de la sociedad moderna. Estos términos muestran contrastes marcados entre el enfoque de capacitación tradicional y las prácticas novedosas. La investigación ha demostrado que cuanto más activos e involucrados se encuentren los docentes en su capacitación profesional, mejores resultados se observarán en los estudiantes. 
Las comunidades o grupos de personas se unen mediante conexiones compartidas que transforman a los individuos en miembros activos de un grupo identificable (Lloyd y Duncan, 2010, p. 61). Las CVA para docentes, por ejemplo, se definen como agrupaciones abiertas y voluntarias de individuos con una preocupación común: la práctica de la docencia. De esta manera, las CVA se valen de diferentes medios de comunicación en línea, tanto sincrónicos como asincrónicos; actúan como una recopilación de ideas y proveen el espacio para compartir retos o situaciones de éxito.

Lloyd y Duncan (2010) afirman que los participantes de una CVA tienen la potestad de decidir si intentan las estrategias en sus propias prácticas de aula o no, en contraste directo con la resistencia de los docentes a usar la investigación o implementar sugerencias de expertos externos o autoridades administrativas, las cuales se incluyen por lo general en las capacitaciones tradicionales. Este cambio se deriva de la motivación personal y las necesidades percibidas en forma individual o colectiva.

Las CVA no difieren mucho de las comunidades físicas (Saborío, s. f.). Sin embargo, ofrecen ventajas como la eliminación de limitaciones de tiempo y distancia, y hacen más accesibles los contactos entre los participantes. También ofrecen una interesante combinación de anonimato e intimidad.

El aprendizaje y el desarrollo profesional pueden ocurrir en toda clase de comunidades, sean estas formales o informales, físicas o virtuales. Sin embargo, las CVA están ganando reconocimiento entre los investigadores como vehículos de creación de conocimiento social y transformación, concluyen Lloyd y Duncan (2010).

En el contexto de una institución educativa, una CVA sería una iniciativa importante si ofrece a los participantes oportunidades de participar en actividades de interés, colaborar para mejorar las prácticas educativas y resolver problemas de la vida cotidiana. Estas actividades promueven la discusión de problemas y desafíos de su propio entorno laboral, el desarrollo profesional y, como resultado, mejoran la calidad de las estrategias metodológicas que se ofrecen al estudiantado.

El proceso clave en las CVA es la colaboración (Lloyd y Duncan, 2010). Las redes colaborativas son particularmente efectivas cuando se mantienen por períodos estables, permitiendo a los educadores aprender y reflejar estos aprendizajes en sus propias prácticas, lo cual repercute 
también en el desempeño académico de aprendizaje de los estudiantes. Al finalizar el tiempo propuesto para el desarrollo del proyecto, se evaluará en conjunto con los participantes, hasta qué punto se alcanzaron estos objetivos.

\section{Inclusión}

La inclusión se define como el proceso de identificar y responder a la diversidad de las necesidades de todos los estudiantes a través de la mayor participación en el aprendizaje, las culturas y las comunidades, y reduciendo la exclusión en educación. Involucra cambios y modificaciones en contenidos, estructuras y estrategias, con una visión común que incluye a todos y con la convicción de que es la responsabilidad del sistema regular educar a todos. Contempla los objetivos de eliminar y superar toda exclusión del derecho humano a la educación, así como promover el acceso, la participación y el éxito en un proceso educativo de calidad.

Los procesos de capacitación y el currículo universitario para docentes regulares rara vez proveen el conocimiento, las habilidades o la práctica necesaria para que los docentes apoyen efectivamente un ambiente inclusivo en las aulas. Como resultado, los docentes de inglés requieren apoyo con actividades de desarrollo profesional para mantener sus prácticas actualizadas y adecuadas a sus contextos particulares.

Los docentes costarricenses de inglés como lengua extranjera tienen el reto de trabajar con estudiantes con y sin NEE en la misma clase, sin contar con bases sólidas en su formación docente para atender efectivamente dichas necesidades. No conocer cómo trabajar en la diversidad puede representar un obstáculo para el planeamiento de oportunidades significativas para desarrollar habilidades adecuadas y necesarias en el aprendizaje comunicativo de un idioma, lo que limitará el ámbito de acción tanto de docentes como de estudiantes.

\section{Constructos teóricos curriculares}

La CVA se enmarca en la importancia de la contextualización curricular, buscando la innovación en diferentes grados: gestión institucional; concepciones sobre el rol docente; capacitación y desarrollo profesional; así como necesidades particulares de los estudiantes en un 
contexto. "Cada vez que los docentes realizan un trabajo reflexivo y colaborativo sobre sus prácticas avanzan en su profesionalidad, innovan sus prácticas y toma de decisiones sobre el currículo" (Pinto, 2012, p. 141). Se considera un elemento decisivo empoderar y posicionar a los docentes como profesionales reflexivos, capaces de tomar decisiones en forma individual o colectiva sobre el currículo que aplican.

Quesada, Cedeño y Zamora (2007) afirman:

El desarrollo de la docencia requiere que los participantes del proceso educativo se apropien de la misión definida por la institución, conozcan y respeten las características de la población meta y realicen una práctica coherente... Ello supone espacios para el desarrollo profesional... (p. 19)

De esta manera, los docentes deben asumir el reto, no solamente desde su papel como facilitadores del aprendizaje, sino comprometidos con su propia actualización profesional. Así responden a las necesidades de la población estudiantil que atienden y del contexto en el cual se desarrolla su labor.

Según Pinto (2012),

Los procesos de planificación a nivel del centro escolar requieren de espacios de autonomía profesional docente en la toma de decisiones curriculares... de tipo colectivo o grupal de los profesores... para el intercambio de experiencias y la reflexión investigativa y crítica de sus prácticas. (p. 141)

Desde esta perspectiva, la institución educativa como unidad principal de investigación e innovación en currículo debe propiciar espacios en torno a una serie de metas comunes, facilitar el diálogo académico, y generar procesos de reflexión que posibiliten el diseño y la implementación de una propuesta académica oportuna, pertinente y flexible (Quesada, Cedeño y Zamora, 2007). Sobre estos supuestos se basa la justificación y formulación de una CVA como espacio de desarrollo profesional para los docentes de inglés con retos comunes.

Por otra parte, según Stenhouse (1984), el conocimiento "no consiste meramente en hechos, sino en hechos de tal modo estructurados que adquieran sentido" (p. 44). Desde el punto de vista de docentes 
reflexivos, esto nos lleva a preguntarnos, ¿adquiere sentido para nuestros estudiantes el conocimiento de una lengua extranjera que pretendemos que pongan en práctica comunicativamente? ¿Adquiere igual sentido para todo el alumnado, bajo las estrategias metodológicas y evaluativas que se están empleando en la actualidad?

Los docentes y estudiantes son los agentes activos del desarrollo curricular. Quesada, Cedeño y Zamora (2007) plantean el desarrollo curricular como un proceso de construcción de conocimiento, como una construcción social a la que estudiantes y docentes imprimen diversos significados y lo transforman con sus aportes.

El conocimiento, tal como lo plantea Stenhouse (1984), también es provisional y cambiante. Esta afirmación es vigente, sobre todo en el área de las lenguas modernas, las cuales se mantienen en constante transformación y evolución. Es preciso que se construyan significados con materiales reales, usando la tecnología que impregna la cotidianidad siempre que el contexto lo permita, y considerando la individualidad del aprendiente para dar sentido a lo que aprende. No se logrará una verdadera habilidad comunicativa si los estudiantes con NEE no logran interiorizar, comprender y dar sentido a lo que aprenden en las aulas de inglés.

Continúa Stenhouse (1984) afirmando que el desarrollo del currículo "ha de basarse en el perfeccionamiento del profesor, que debe promocionar y acentuar su profesionalismo" (p. 54). Se busca entonces que los docentes fortalezcan su práctica comprobando ideas, de forma reflexiva y sistemática, y proponiendo en forma espontánea pero comprometida estrategias de enseñanza innovadoras para fortalecer el aprendizaje. Esta idea de currículo flexible, adaptable, que involucra al docente y sobre el que se reflexiona constantemente es la base para la construcción de la CVA. "Si el conocimiento ha de ser considerado como un recurso y un sistema abierto, y no como una imposición por parte de aquellos que lo poseen, es necesario desarrollar nuevos estilos de enseñanza" (Stenhouse, 1984, p. 63).

No es tan importante el hecho de que terceras personas estudien la labor de los docentes en las aulas para determinar si contribuye al aprendizaje significativo de todos los aprendientes: necesitan estudiarla ellos mismos, convertirse en investigadores de su propia situación docente. Stenhouse (1984) propone tres características de un profesional en educación: "capacidad para un autodesarrollo profesional autónomo..., 
el estudio de la labor de otros profesores y la comprobación de ideas mediante procedimientos de investigación" (p. 197).

Es así como una CVA contribuye a la autorreflexión de cada uno de los participantes, a la interacción entre los educadores de la misma institución o campo de estudio, y a la deliberación sobre las estrategias o retos propuestos por los colegas en su práctica cotidiana. Una CVA así planteada contribuye al crecimiento profesional a través de la reflexión, la colaboración y la investigación activa de los docentes en sus contextos particulares, haciendo valiosos cada uno de los aportes y transformando los dilemas de la práctica docente cotidiana en retos para el docente y sus pares.

En cuanto a los componentes del diseño curricular propuestos por Quesada, Cedeño y Zamora (2007), tanto la justificación, la fundamentación, el perfil de salida, los objetivos y la estructura curricular están claramente definidos en el Programa de Estudio 2005 Inglés Educación Diversificada. Sin embargo, el hecho de que se defina teóricamente el enfoque comunicativo y la aplicación de las inteligencias múltiples según Oxford en como sustentos teóricos para las estrategias metodológicas no significa que ellos sean utilizados masivamente en las aulas, al enfrentarse los docentes al final del proceso a una estrategia evaluativa basada únicamente en la comprensión lectora. Por otra parte, los objetivos propuestos como parte del programa y que finalmente integran el perfil de salida del estudiante se evalúan en forma generalizada. Es aquí donde los docentes deben intervenir, asumiendo su rol activo e innovador, para lograr los cambios necesarios por medio de la aplicación de estrategias metodológicas coherentes con la población de aprendientes y las demandas del entorno.

El poder de un profesor aislado es limitado. Sin sus esfuerzos jamás se puede lograr la mejora de las escuelas; pero los trabajos individuales son ineficaces si no están coordinados y apoyados. La unidad primaria de coordinación y apoyo es la escuela. (Stenhouse, 1984, p. 223)

\section{Propuesta de Contextualización Curricular}

El objetivo principal de la investigación es diseñar, construir e implementar un sitio web para formar una CVA basada en diferentes 
estrategias y técnicas para enseñar a los estudiantes de inglés que presentan NEE. El sitio web que se propone buscará funcionar como un esfuerzo colaborativo de desarrollo profesional, por medio de la investigación-acción participativa, construido por y para docentes de inglés, con el fin de compartir estrategias para ajustar sus prácticas educativas a la atención de estudiantes de todas las habilidades en sus aulas. Se busca aprender colectivamente, para mejorar la propia práctica educativa e incidir positivamente en los aprendizajes de todos los estudiantes.

Las barreras existentes en la actualidad para que el educador se capacite y crezca profesionalmente también limitan la calidad de la enseñanza que ofrecen a sus estudiantes, lo que da gran auge a la creación de CVAs. En ellas, el aprendizaje de los estudiantes va unido y es potenciado por el desarrollo profesional del docente.

Según Saborío (s. f.), "en un principio dichas comunidades se originaron con fines comerciales, recreativos o culturales, en las cuales se aprovechaba el espacio para que los miembros interactúen a conveniencia.... Posteriormente, las comunidades virtuales empiezan a adaptarse como una oportunidad a servicio del aprendizaje" (p. 3). Esta es una alternativa que se dirige a los docentes con el fin de descubrir, discutir y desarrollar destrezas en el área de las prácticas inclusivas, integradas con las habilidades específicas de la asignatura y los estándares curriculares. Como consecuencia, se busca mejorar la calidad de la educación que se ofrece a los estudiantes.

Los docentes que laboran en la institución son conscientes de la necesidad de capacitación en esta área, debido a la inexistencia de una carrera universitaria o énfasis que los prepare, tanto en la enseñanza del inglés como en la atención a la diversidad. Por esta razón, se prevé que la CVA funcione como un foco de diagnóstico de necesidades docentes con base en los retos que enfrentan en sus aulas; estimule el desarrollo de propuestas surgidas de la investigación, tanto externa como interna; y finalmente logre articular una evaluación formativa del impacto de esta participación colaborativa en la calidad de educación que se imparte a los estudiantes. El proceso de evaluación formativa al final del proceso responderá dichas interrogantes.

La CVA contempla recursos sincrónicos y asincrónicos de comunicación en línea, como blogs, podcasts, videos, información en forma de archivos descargables, gráficos y diapositivas, así como un perfil de Twitter con las últimas novedades del sitio web. 


\section{Conclusiones}

¿Por qué una CVA puede servir para contextualizar el currículo?

En el caso particular de la enseñanza del inglés como lengua extranjera, es muy marcada la influencia ideológica y política de su puesta en marcha. Los gobiernos que han implementado la enseñanza del inglés en las aulas costarricenses desde la educación primaria han promovido la provisión de experiencias culturales y conocimientos relevantes en esta área bajo la firme creencia de que en el futuro estas facilitarán la apertura de oportunidades de acceso laboral, que resuelvan problemas de inequidad. MEP (2003) señala que, "la educación debe contribuir a reducir la brecha socioeconómica, proporcionándole a los individuos las oportunidades adecuadas para integrarse en situaciones diarias de resolución de problemas" (p. 15).

Siendo el currículo una construcción social, de Alba (1991) lo define como una síntesis de elementos "culturales... conocimientos, valores, creencias, costumbres y hábitos, que conforman una determinada propuesta político-educativa" (p. 39). Un diseño curricular así planteado busca la generación de respuestas originales y contextualizadas a problemas específicos y con estudiantes diversos. Los agentes encargados de diseñar estas propuestas deben tomar decisiones de tipo "pedagógico y metodológico, rasgos del perfil del profesor y del alumno; hábitos de enseñanza y de aprendizaje, factores espacio-temporales, objetivos de enseñanza, motivación" (Núñez, 2008, p. 2), entre otros. El programa de inglés del MEP (2003) fue escrito...

dentro de los principios establecidos por la Constitución Política de Costa Rica, la Ley de Educación y la Política Educativa "Hacia el Siglo XXI" para ayudar a los estudiantes a enfrentar la vida y las situaciones laborales, las que requieren un dominio promedio del inglés, con el deseo de que esta preparación les permita participar activamente en los retos de la economía global, para el beneficio del país (p. 14).

De la descripción anterior también podemos deducir, la importancia que se le da a la enseñanza y el aprendizaje del inglés en Costa Rica, los fines para los cuales fueron diseñados estos programas y los conocimientos que espera establecer esta propuesta político-educativa. 
Estas son aspiraciones del sistema educativo costarricense, que deben ser contextualizadas en cada institución educativa para que representen aprendizajes significativos para sus estudiantes.

Por otra parte, de Alba (1991) también señala la importancia de la función social de la educación. Se ha superado la visión de que el currículo funge como reproductor del orden social imperante, para privilegiar la reflexión y elaboración conceptual, aunque la autora señala que la óptica teórica reproduccionista es la que ha imperado en el diseño curricular.

Podemos aplicar estos conceptos al currículo actual de la enseñanza del inglés, donde según el MEP (2003): "El enfoque comunicativo provee las bases para la metodología usada en el aula de inglés" (p. 23). Este enfoque se originó hacia 1960 a partir de un movimiento de lingüistas británicos como reacción a los modelos que parten de la gramática. Tanto los proponentes de Gran Bretaña como de los Estados Unidos sugieren que lo más importante es desarrollar la competencia comunicativa y abrir procesos de aprendizaje para la enseñanza de las cuatro habilidades lingüísticas básicas. (Domian, Fonseca, Lara y Rodríguez, 2011). El programa también cita las definiciones de Rebeca Oxford sobre estrategias de aprendizaje dentro de sus definiciones.

¿Se adaptan verdaderamente el enfoque comunicativo y las estrategias de aprendizaje en las clases de inglés dentro del contexto costarricense? Si tomamos en cuenta la estrategia evaluativa final del programa de inglés en la Educación Diversificada, con una prueba de bachillerato basada en la comprensión de lectura, no podríamos relacionar los conceptos del enfoque comunicativo ni aplicar las estrategias de aprendizaje de Oxford para enfrentar una prueba de esta modalidad. Por lo tanto, es entendible por qué las estrategias metodológicas de aula busquen más obtener respuestas en este sentido y no estimulen las habilidades comunicativas.

Sin embargo, unas habilidades no excluyen a las otras; se requiere criticidad y desarrollo profesional con conocimientos teóricos y prácticos para estimular el aprendizaje del inglés en todas sus dimensiones, incluyendo a todos los estudiantes, para alcanzar el éxito en la vida académica y la inclusión laboral posterior. Lo anterior también empodera a los docentes para innovar el currículo y ser protagonista, junto con el alumnado, de las estrategias que aplique en el aula. 
No sólo los aspectos estructurales-formales de un currículo son la prioridad. De Alba (1991), señala que "el desarrollo procesal-práctico de un currículo es fundamental para comprender su constitución determinante como su devenir en las instituciones escolares concretas" (p. 43). Es en este punto donde las particularidades de cada región educativa, circuito escolar e incluso de cada institución en su singularidad se expresan en la aplicación del currículo. Tener en cuenta los distintos contextos en los que cada escuela se ha desarrollado, las condiciones en las que los docentes enseñan, su formación profesional, las particularidades de su enseñanza y las características de sus estudiantes (incluyendo las NEE que presenta su población) permitirá que la toma de decisiones curriculares y organizacionales promueva una escuela para todos.

Estas ideas de De Alba son apoyadas por el MEP (2003) cuando afirma que, "la investigación educativa a niveles nacional, provincial, regional e institucional asegurará una implementación más sistemática de la política, desde la práctica educativa diaria en las aulas hasta la toma de decisiones administrativas. El diseño del programa promueve la interacción participativa y su adaptación" (p. 16). ¿Qué tanto de este discurso es parte del currículo práctico de las aulas de inglés? ¿Es realmente participativo y adaptable el currículo? ¿Asumen los docentes de inglés su responsabilidad al implementar variaciones en las políticas educativas emanadas por el MEP, sobre todo cuando deben atender estudiantes con NEE en sus aulas?

En este sentido práctico, dinámico y reflexivo del currículo se enmarca la propuesta didáctica de una CVA, ya que se desea aplicar una personalización de las estrategias de aprendizaje para atender mejor las NEE del estudiantado y la inclusión en general. Se puede afirmar que no existe un currículo único, no solo porque los objetivos de aprendizaje en su aplicación varían de un sujeto a otro, sino porque "los resultados nunca son idénticos en los distintos sujetos, aun cuando éstos sean sometidos al mismo proceso de enseñanza-aprendizaje y su nivel de motivación sea, supuestamente, el mismo" (Núñez París, 2008, p. 23).

En la actualidad, se dan varias fuerzas que afectan al currículo, como los avances en el comercio global, los cuales abren espacio a las empresas de carácter transnacional instaladas en los países del Tercer Mundo, que a su vez generan un cambio en las exigencias de capacitación que se hacen desde el aparato productivo. Esto también se refleja en el plan de estudios de inglés de Educación Diversificada. MEP 
(2003) indica que "alcanzar la sostenibilidad en la producción y la economía en general representa un reto para la educación. El país necesita personas más calificadas para incrementar la productividad y mejorar el espíritu de competitividad. Además, existe la necesidad de integrar el país más eficientemente en la economía global” (p. 15).

Por otra parte, la comunicación en el mundo contemporáneo se ha globalizado: medios masivos de comunicación juegan un papel importante en la transmisión, producción y reproducción cultural e ideológica. Ya no lo es solamente la televisión, sino también a través de Internet y el acceso a la telefonía celular, las exigencias de la educación constante y el aprender a aprender surgen con más fuerza.

El sujeto social se caracteriza por tener conciencia histórica por ser parte de un grupo que suscribe un determinado proyecto social, y sus acciones se inscriben en una determinada direccionalidad social. Es entonces función del profesor reflexionar y analizar los diferentes tipos de currículo que subyacen en la enseñanza de las lenguas modernas, teniendo como referencia "los elementos constitutivos que cada modelo de diseño curricular recoge, a saber: el qué, el cómo y el cuándo se aprende, siendo estos tres factores los que diferencian los respectivos tipos de currículo" (Núñez París, 2008, p. 26).

Un objetivo fundamental de la CVA es crear para los docentes de inglés un espacio reflexivo, donde el currículo se encuentra en transformación constante, al tomar en cuenta las habilidades de los estudiantes, el contexto globalizado, la influencia de la tecnología y la información en la vida cotidiana, así como las estrategias de aprendizaje y evaluación que, al ser modificadas, generan aprendizajes más significativos y duraderos.

Si bien es cierto, la política educativa busca vincular la educación con el aparato productivo, existe la necesidad en las aulas de construir alternativas colectivas que respondan a nuestra condición latinoamericana, con las características particulares que encontramos en cada institución, en cada aula, en cada estudiante y en cada docente, con el fin de articular, con sentido propio, las prácticas educativas con las demandas del sistema educativo. La CVA se suscribe a estas ideas en cuanto pretende ser un aporte que permita a los docentes, junto con los otros y en el contexto de un análisis público y colectivo, asumirse como sujetos innovadores de la determinación curricular. 
El sujeto será activo si es capaz de distinguir lo viable de lo deseable, y si su acción se inscribe en una concepción del futuro como horizonte de acciones posibles.

\section{Referencias bibliográficas}

Bolívar Ruano, M. R. (s. f.). Comunidades profesionales de aprendizaje. Instrumentos de diagnóstico y evaluación. Granada, España. Descargado de http:/hum386.ugr.es/media/grupos/HUM386/ $\mathrm{cms} /$ comunidades $\% 20$ profesionales $\% 20 \mathrm{de} \% 20$ aprendizaje $\% 20$ Instrumentos_Rosel\%20Bolivar.pdf

De Alba, A. (1991). Curriculum: Crisis, mito y perspectivas. México, DF: Universidad Autónoma de México.

Domian, C., Fonseca, H., Lara, R. \& Rodríguez, S. (2011). La enseñanza comunicativa del inglés en el ciclo diversificado en Costa Rica: imágenes de algunas realidades. Letras, 47, 11-34.

Lloyd, M. y Duncan, J. (2010). Changing the Metaphor: The Potential of Online Communities in Teacher Professional Development. En O. Lindberg y A. Olofsson (Eds.), Online Learning Communities and Teacher Professional Development. (pp. 60-76). Hershey, PA: Information Science Reference

Lewin, K. (1999). Personal adjustment and group belongingness. In M. Gold (Ed.), A Kurt Lewin reader: The complete social scientist (pp. 327-332). Washington, DC: American Psychological Association. (Original work published 1941)

Ministerio de Educación Pública. (2005). Programa de Estudio. Inglés, Educación Diversificada. San José, CR: Imprenta Nacional.

Núñez París, F. (2008). "Teoría del currículo y didáctica de las lenguas extranjeras". Revista Nebrija de Lingüistica Aplicada a la Enseñanza de Lenguas, 4, p. 1-30. Descargado de http://www. nebrija.com/revista-linguistica/files/articulosPDF/articulo_531f2c7ea2c17.pdf

Paz, G., \& Suárez, P. (2010). Diseño curricular para la educación secundaria. Inglés. Buenos Aires, Argentina: Dirección General de Cultura y Educación.

Pinto, R. (2012). Principios filosóficos y epistemológicos del ser docente. San José, CR: Coordinación Educativa y Cultural Centroamericana. 
Quesada, Cedeño y Zamora. (2007). El diseño curricular en los planes de estudio: aspectos teóricos y guía metodológica. Heredia, CR: EUNA.

Richards, J. C. y Farrell, T. S. (2005). Professional Development for Language Teachers. Strategies for Teacher Learning. Cambridge, MA: Cambridge University Press.

Saborío, S. (s.f.) La comunidad de aprendizaje virtual aplicada a la pedagogía. San José: Universidad Estatal a Distancia. Retrieved from http://redes-y-comunidades.wikispaces.com/file/view/SILVIA_Ensayo.pdf

Stenhouse, L. (1984). Investigación y desarrollo del currículo. Madrid: Ediciones Morata. 